\title{
The Kodaira Problem for Kähler Spaces with Vanishing First Chern Class
}

\author{
Patrick Graf ${ }^{1}$ and Martin Schwald ${ }^{2}$ \\ ${ }^{1}$ Lehrstuhl für Mathematik I, Universität Bayreuth, 95440 Bayreuth, Germany; E-mail: patrick.graf@uni-bayreuth.de \\ URL: www.graficland.uni-bayreuth.de. \\ ${ }^{2}$ Fakultät für Mathematik, Universität Duisburg-Essen, 45117 Essen, Germany; E-mail: martin.schwald@uni-due.de \\ URL: www.esaga.uni-due.de/martin.schwald/.
}

Received: 28 May 2020; Accepted: 25 November 2020

2010 Mathematics Subject Classification: 32J27, 14E30, 32G05

\begin{abstract}
Let $X$ be a normal compact Kähler space with klt singularities and torsion canonical bundle. We show that $X$ admits arbitrarily small deformations that are projective varieties if its locally trivial deformation space is smooth. We then prove that this unobstructedness assumption holds in at least three cases: if $X$ has toroidal singularities, if $X$ has finite quotient singularities and if the cohomology group $\mathrm{H}^{2}\left(X, \mathscr{T}_{X}\right)$ vanishes.
\end{abstract}

\section{Contents}

1 Introduction

2 Basic facts and definitions

3 A criterion for algebraic approximability 7

4 Surjectivity of cupping with a Kähler class $r$

5 Locally trivial unobstructedness

\section{Introduction}

\section{A. The Kodaira problem}

A compact complex manifold is projective if and only if it admits a Kähler form whose cohomology class is rational. Thus it is a natural question to ask whether any compact Kähler manifold $X$ can be made projective by a (small) deformation of its complex structure. This question is made precise by the notion of an algebraic approximation of $X$; see Definition 2.2. The problem was already studied by Kodaira, who proved that every compact Kähler surface can be deformed to an algebraic surface [Kod63], and it has been known as the Kodaira problem ever since.

Recent progress gives a complete positive answer to the Kodaira problem in dimension 3 [Gra18, CHL19, Lin18a]. In addition, Voisin provided examples showing that starting in dimension 4 there are compact Kähler manifolds without algebraic approximations [Voi04, Voi06]. Voisin's examples show how blowups can destroy the existence of algebraic approximations. However, having the philosophy of the minimal model program (MMP) in mind, this raises the question whether the Kodaira problem

(C) The Author(s), 2021. Published by Cambridge University Press. This is an Open Access article, distributed under the terms of the Creative Commons Attribution licence (http://creativecommons.org/licenses/by/4.0/), which permits unrestricted re-use, distribution, and reproduction in any medium, provided the original work is properly cited. 
for $X$ should instead be posed for a (possibly singular) minimal model of $X$. In this spirit, the following conjecture of Peternell becomes of interest.

Conjecture 1.1 (Peternell). Let $X$ be a minimal compact Kähler space. Then $X$ admits an algebraic approximation.

Related positive results in higher dimensions are still scarce [Lin18b], and from the MMP point of view no natural extension of this conjecture to the case $\kappa(X)=-\infty$ is expected to hold [GS20].

\section{B. The case of Kodaira dimension 0}

When studying the Kodaira problem in higher dimensions, it is natural to aim at the case of Kodaira dimension 0 first. For a compact Kähler manifold $X$ with trivial canonical bundle $K_{X} \sim 0$, the existence of an algebraic approximation can be proved by a combination of three well-known results:

(A) The BTT (Bogomolov-Tian-Todorov) theorem states that the deformations of $X$ are unobstructed; that is, the semi-universal deformation space $\operatorname{Def}(X)$ is smooth [Bog78, Tia87, Tod89].

(B) By the Green-Voisin criterion it is then sufficient to prove that cupping with a Kähler class $[\omega] \epsilon$ $\mathrm{H}^{1}\left(X, \Omega_{X}^{1}\right)$ induces a surjection $\mathrm{H}^{1}\left(X, \mathscr{T}_{X}\right) \longrightarrow \mathrm{H}^{2}\left(X, \mathcal{O}_{X}\right)$ [Voi03, Prop. 5.20].

(C) Using the assumption $K_{X} \sim 0$, the latter surjectivity is easily reduced to the hard Lefschetz theorem.

More generally, if $X$ is a compact Kähler manifold with torsion canonical bundle $K_{X} \sim{ }_{\mathbb{Q}} 0$, one passes to the index 1 cover $X_{1} \rightarrow X$, on which the canonical bundle becomes trivial. An algebraic approximation of $X_{1}$ then induces an algebraic approximation of $X$. With Peternell's conjecture in mind, in the present work we study the case of a general minimal compact Kähler space $X$ of Kodaira dimension 0 by extending the above line of reasoning.

\section{C. Main results}

If the conjectures of the Kähler MMP hold, studying Conjecture 1.1 for Kodaira dimension 0 means considering normal compact Kähler spaces $X$ with klt singularities such that the canonical class is torsion, $K_{X} \sim \mathbb{Q} 0 .{ }^{1}$ As our first main result we establish that steps $(\mathrm{B})$ and $(\mathrm{C})$ above indeed generalize to this setting if one considers not all of the deformations of a given $X$ but only the locally trivial ones; see Definition 2.3. This is done in Theorems 3.1 and 4.1, respectively. Taken together, they yield the following.

Theorem 1.2 (Algebraic approximation for unobstructed spaces). Let X be a normal compact Kähler space with klt singularities and $K_{X} \sim \mathbb{Q} 0$. If the base of the semi-universal locally trivial deformation $\mathfrak{X} \rightarrow \operatorname{Def}^{\mathrm{lt}}(X)$ is smooth, then $\mathfrak{X} \rightarrow \operatorname{Def}^{\mathrm{lt}}(X)$ is a strong algebraic approximation of $X$.

In light of this result, it becomes important to study step (A); that is, to prove a BTT theorem in our setting. The problem is that though there do exist some singular versions of BTT for 3-folds and under other strong hypotheses, it is also well-known that the full result becomes invalid in the presence of singularities: Examples by Gross show that for $n \geq 3$ the deformation space of a Calabi-Yau $n$-fold with canonical singularities need not even be locally irreducible [Gro97]. Gross's examples, however, are easily seen not to be locally trivial. In particular, in the literature there is no answer to the following question.

Question 1.3 (Locally trivial BTT). Is $\operatorname{Def}^{\mathrm{lt}}(X)$ smooth for any normal compact Kähler space with klt singularities and $K_{X} \sim \mathbb{Q} 0$ ?

\footnotetext{
${ }^{1}$ To be technically precise, we require that a reflexive tensor power of the canonical sheaf $\omega_{X}$ is trivial; that is, $\omega_{X}^{[m]} \cong \mathcal{O}_{X}$ for some $m>0$.
} 
For primitive symplectic varieties, the answer to this question is yes due to Bakker and Lehn [BL19, Thm. 4.11]. We do not know whether the answer is always yes, but we do prove that Question 1.3 has a positive solution in the following two cases. The first one is a generalisation of the well-known unobstructedness criterion of Kodaira-Nirenberg-Spencer (KNS) [KNS58] to locally trivial deformations.

Theorem 1.4 (Locally trivial KNS theorem). Let $X$ be a normal compact complex space with $\mathrm{H}^{2}\left(X, \mathscr{T}_{X}\right)=0$. Then $\operatorname{Def}^{\mathrm{lt}}(X)$ is smooth.

Remark. A stronger statement than Theorem 1.4 is that $\mathrm{H}^{2}\left(X, \mathscr{T}_{X}\right)$ is an obstruction space for the locally trivial deformation functor $D_{X}^{\text {lt }}$ of $X$. This means that for every sequence $0 \rightarrow J \rightarrow A^{\prime} \rightarrow A \rightarrow 0$ of local Artinian $\mathbb{C}$-algebras with residue field $\mathbb{C}$ and such that $\mathfrak{m}_{A^{\prime}} J=0$, there is an exact sequence of pointed sets $D_{X}^{\mathrm{lt}}\left(A^{\prime}\right) \rightarrow D_{X}^{\mathrm{lt}}(A) \rightarrow \mathrm{H}^{2}\left(X, \mathscr{T}_{X}\right) \otimes J$. This is claimed (in the algebraic case) in [Ser06, Prop. 2.4.6], but the proof contains a gap: In order to extend a local automorphism of a deformation of $X$ over $A$ to $A^{\prime}$, the infinitesimal lifting property is invoked. This, however, is only possible if $X$ is smooth. Our Theorem 1.4 weakly fills this gap.

The second case where we can prove unobstructedness is when the Hodge theory of reflexive differential forms on $X$ behaves nicely. More precisely, denote by $\Omega_{X}^{[\bullet]}=j_{*} \Omega_{U}^{\bullet}$ the complex of reflexive differential forms on $X$, where $j: U \rightarrow X$ is the inclusion of the smooth locus. Then we require two conditions: Firstly, the natural map $\mathbb{C}_{X} \rightarrow \Omega_{X}^{[\bullet]}$ should be a quasi-isomorphism. Equivalently, the Poincaré lemma for reflexive differential forms should hold on $X$ : Every closed reflexive form of degree $\geq 1$ is locally exact. Under this assumption there is a Hodge to de Rham spectral sequence, coming from the filtration bete on $\Omega_{X}^{[\bullet]}$ :

$$
E_{1}^{p, q}=\mathrm{H}^{q}\left(X, \Omega_{X}^{[p]}\right) \Longrightarrow \mathrm{H}^{p+q}(X, \mathbb{C}) .
$$

The second requirement is that this spectral sequence degenerate on the first page in degree $n=\operatorname{dim} X$. That is, we require the differentials $d_{r}^{p, q}$ to vanish for all $r \geq 1$ and $p+q=n$.

Theorem 1.5. Let $X$ be an n-dimensional normal compact Kähler space with rational singularities and trivial canonical class $K_{X} \sim 0$ such that the reflexive Poincaré lemma holds on $X$ and (1.4.1) degenerates at $E_{1}$ in degree $n$. Then $\operatorname{Def}^{\mathrm{lt}}(X)$ is smooth.

The assumptions in Theorem 1.5 are very strong: Even for klt singularities, the reflexive Poincaré lemma can fail in degree at least 2, and if it holds, the spectral sequence (1.4.1) need not degenerate on the first page [Jör14, Prop. 1.6(b)]. However, the assumptions do hold in at least two interesting cases:

$\circ$ if $X$ has only (finite) quotient singularities [Ste77], and

$\circ$ if $X$ is a toroidal complex space; that is, it is locally analytically isomorphic to the germ of a toric variety at a torus fixed point [Dan91].

We deduce the following corollary.

Corollary 1.6 (Locally trivial BTT for orbifold/toroidal singularities). Let $X$ be a normal compact Kähler space with either only quotient or only toroidal singularities and $K_{X} \sim \mathbb{Q} 0$. Then $\operatorname{Def}^{\text {lt }}(X)$ is smooth.

Summing up Theorems 1.2 and 1.4 and Corollary 1.6, we have the following positive partial answer to Peternell's conjecture:

Corollary 1.7. Let $X$ be a normal compact Kähler space with $K_{X} \sim_{\mathbb{Q}} 0$. Assume any of the following:

(1.7.1) $X$ has only quotient singularities.

(1.7.2) $X$ has only toroidal singularities.

(1.7.3) $X$ is klt and the cohomology group $\mathrm{H}^{2}\left(X, \mathscr{T}_{X}\right)$ vanishes.

Then $X$ admits a strong algebraic approximation. 
Remark. In the orbifold case (1.7.1), the analogue of the Beauville-Bogomolov decomposition for $X$ is already known [Cam04, Thm. 6.4]. It is tempting to try to use this splitting result in order to obtain an algebraic approximation. Our approach is completely different, because it does not rely on any kind of structure theorem.

\section{D. Recent progress}

Since this article was first posted on the arXiv, substantial progress has been made on the questions discussed herein. Building on our Theorems 3.1 and 4.1 and on further work of the first-named author [CGGN20], Conjecture 1.1 and the Beauville-Bogomolov decomposition have been established for klt Kähler spaces with $\mathrm{c}_{1}=0$ [BGL20, Gra21]. This was done by proving a weak version of the BTT unobstructedness theorem that only deals with deformations in the 'symplectic direction'. In general, the answer to Question 1.3 remains unknown and our Corollary 1.6 remains one of the few partial results available.

\section{Basic facts and definitions}

\section{Complex spaces}

All complex spaces are assumed to be separated and connected, unless otherwise stated. An open subspace of a complex space is called big if its complement is analytic and has codimension at least 2.

\section{Deformation theory}

We collect some basic definitions and facts from deformation theory.

Definition 2.1 (Deformations of complex spaces). A deformation of a reduced compact complex space $X$ is a proper flat morphism $\pi: \mathfrak{X} \rightarrow(S, 0)$ from a (not necessarily reduced) complex space $\mathfrak{X}$ to a complex space germ $(S, 0)$, equipped with a fixed isomorphism $X_{0} \cong X$, where we write $X_{s}:=\pi^{-1}(s)$ for the fibre over any $s \in S$. We usually suppress both the base point $0 \in S$ and the choice of isomorphism from notation.

If $S=\operatorname{Spec} A$ for some local Artinian $\mathbb{C}$-algebra $A$ with residue field $\mathbb{C}$, the deformation $\mathfrak{X} \rightarrow S$ is called infinitesimal.

Definition 2.2 (Algebraic approximations). Let $X$ be a compact complex space and $\pi: \mathfrak{X} \rightarrow S$ a deformation of $X$. Consider the set of projective fibres

$$
S^{\text {alg }}:=\left\{s \in S \mid \mathfrak{X}_{s} \text { is projective }\right\} \subset S
$$

and its closure $\overline{S^{\text {alg }}} \subset S$. We say that $\mathfrak{X} \rightarrow S$ is an algebraic approximation of $X$ if $0 \in \overline{S^{\text {alg }}}$. We say that $\mathfrak{X} \rightarrow S$ is a strong algebraic approximation of $X$ if $\overline{S^{\text {alg }}}=S$ as germs - that is, $S^{\text {alg }}-$ is dense near $0 \in S$.

Definition 2.3 (Locally trivial deformations). A deformation $\pi: \mathfrak{X} \rightarrow S$ is called locally trivial if for every $x \in \mathfrak{X}_{0}$ there exist open subsets $0 \in S^{\circ} \subset S$ and $x \in U \subset \pi^{-1}\left(S^{\circ}\right)$ and an isomorphism

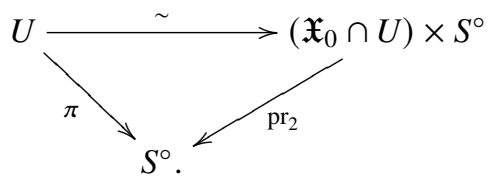

Any compact complex space $X$ admits a semi-universal locally trivial deformation $\mathfrak{X} \rightarrow \operatorname{Def}{ }^{l t}(X)$, and $\operatorname{Def}^{\text {lt }}(X)$ is a closed subspace of the semi-universal deformation space $\operatorname{Def}(X)$. This is proved in [FK87, Cor. 0.3]. 


\section{Resolution of singularities}

A resolution of singularities of a complex space $X$ is a proper bimeromorphic morphism $f: \widetilde{X} \rightarrow X$, where $\widetilde{X}$ is a complex manifold. We say that the resolution is projective if $f$ is a projective morphism. In this case, if $X$ is projective (respectively compact Kähler) then so is $\widetilde{X}$. A resolution is said to be strong if it is a biholomorphism over the smooth locus of $X$.

Theorem 2.4 (Functorial resolutions). There exists a resolution functor that assigns to any complex space $X$ a strong projective resolution $\pi_{X}: \mathcal{R}(X) \rightarrow X$, such that $\mathcal{R}$ commutes with smooth maps in the following sense: For any smooth morphism $f: W \rightarrow X$, there is a unique smooth morphism $\mathcal{R}(f): \mathcal{R}(W) \rightarrow \mathcal{R}(X)$ such that the following diagram is a fibre product square:

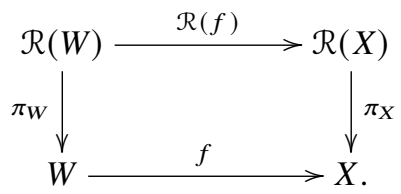

Proof. See [Kol07, Thm. 3.45].

Definition 2.5 (Simultaneous resolutions). Let $\mathfrak{X} \rightarrow S$ be a deformation of $X$. A simultaneous resolution of $\mathfrak{X} \rightarrow S$ is a proper bimeromorphic map $\mathfrak{Y} \rightarrow \mathfrak{X}$ such that the composition $\mathfrak{Y} \rightarrow S$ is a smooth morphism.

Unlike the general case, simultaneous resolutions do exist for locally trivial deformations. This is proved in [BL19, Lemma 4.6], but see also [GS20, Lemma 4.2]. If the deformation in question is locally given as a product $U \times S$ with $U \subset X$ open, then on the preimage sets, $\mathfrak{Y}$ will be given by $\mathcal{R}(U) \times S$. In particular, the restriction of $\mathfrak{Y} \rightarrow \mathfrak{X}$ to the central fibre is the functorial resolution $Y=\mathcal{R}(X) \rightarrow X$, and $\mathfrak{Y} \rightarrow S$ is a (locally trivial) deformation of $Y$.

\section{Reflexive sheaves on nonreduced spaces}

For the rest of this section, we fix the following setting: $X$ is a normal compact complex space and $\pi: \mathfrak{X} \rightarrow S=\operatorname{Spec} A$ is an infinitesimal deformation of $X$.

Lemma 2.6. The sheaf $\mathcal{O}_{\mathfrak{X}}$ is torsion free and normal; that is, for any open subset $U \subset \mathfrak{X}$ and any analytic subset $Z \subset U$, the following holds:

(2.6.1) The restriction map $\Gamma\left(U, \mathcal{O}_{\mathfrak{x}}\right) \rightarrow \Gamma\left(U \backslash Z, \mathcal{O}_{\mathfrak{X}}\right)$ is injective.

(2.6.2) If $\operatorname{codim}_{\mathfrak{X}}(Z) \geq 2$, then the above restriction map is bijective.

Proof. Let $\mathrm{m} \subset A$ be the maximal ideal and set $S_{n}=\operatorname{Spec} A_{n}$, where $A_{n}:=A / \mathrm{m}^{n+1}$. We will prove by induction on $n$ that the claims hold for the base change $\mathfrak{X}_{n} \rightarrow S_{n}$, for every $n \in \mathbb{N}$. This is sufficient because $A$ is Artinian and so $A_{n}=A$ for $n \gg 0$.

For $n=0$, the statement is true because $\mathfrak{X}_{0}=X$ is assumed to be normal. For the inductive step, assume the statement for $\mathfrak{X}_{n-1}$ and consider the short exact sequence

$$
0 \longrightarrow \mathrm{m}^{n} / \mathrm{m}^{n+1} \longrightarrow A / \mathrm{m}^{n+1} \longrightarrow A / \mathfrak{m}^{n} \longrightarrow 0
$$

Note that $\mathfrak{m}^{n} / \mathfrak{m}^{n+1}$ is annihilated by $\mathfrak{m}$; hence it is in fact an $A / \mathfrak{m}$-module (that is, a $\mathbb{C}$-vector space, say of dimension $N$ ) and its $A$-module structure is obtained by pullback along the natural map $A \rightarrow \mathbb{C}$. Thus, pulling back sequence (2.6.3) to $\mathfrak{X}$, by flatness we obtain an exact sequence

$$
0 \longrightarrow \mathcal{O}_{X}^{\oplus N} \longrightarrow \mathcal{O}_{\mathfrak{X}_{n}} \longrightarrow \mathcal{O}_{\mathfrak{X}_{n-1}} \longrightarrow 0 .
$$


Torsion-freeness (2.6.1) follows easily from this by a diagram chase. For normality (2.6.2), note that extendability over $Z$ is a local property because of torsion-freeness. We may thus assume that $\mathfrak{X}$ is Stein. In this case, the claim again follows from a diagram chase.

It follows from Lemma 2.6 that $\mathscr{F}^{\vee}:=\mathscr{H} o m\left(\mathscr{F}, \mathcal{O}_{\mathfrak{X}}\right)$ is torsion free and normal for any coherent sheaf $\mathscr{F}$ on $\mathfrak{X}$. In particular, if $\mathscr{F}$ is reflexive in the sense that the natural map $\mathscr{F} \rightarrow \mathscr{F}^{v v}$ to the double dual is an isomorphism, then $\mathscr{F}$ is torsion free and normal. Conversely, if $\mathscr{F}$ is torsion free and normal and locally free on a big open subset $\mathfrak{U} \subset \mathfrak{X}$, then it is reflexive.

Now let $\mathscr{F}$ be coherent and locally free on a big open subset $\mathfrak{U} \subset \mathfrak{X}$ (but not necessarily torsion free and normal). Then the double dual of the natural map $\mathscr{F} \rightarrow i_{*} i^{*} \mathscr{F}$ is an isomorphism, where $i: \mathfrak{U} \hookrightarrow \mathfrak{X}$ denotes the inclusion. In other words, $\mathscr{F}^{v v} \cong i_{*} i^{*} \mathscr{F}$ for such a sheaf.

We apply the above observations to various sheaves of Kähler differentials. Let $\Omega_{\mathfrak{X} / S}^{1}$ be the sheaf of relative differentials of the morphism $\pi$, and set $\Omega_{\mathfrak{X} / S}^{p}:=\bigwedge^{p} \Omega_{\mathfrak{X} / S}^{1}$ for any integer $p \geq 0$. The smooth locus $i: \mathfrak{U} \hookrightarrow \mathfrak{X}$ of $\pi$ is a big open subset, and $i^{*} \Omega_{\mathfrak{X} / S}^{1}=\Omega_{\mathfrak{U} / S}^{1}$ is locally free. We define the sheaf of relative reflexive $p$-forms to be $\Omega_{\mathfrak{X} / S}^{[p]}:=\left(\Omega_{\mathfrak{X} / S}^{p}\right)^{\downarrow}=i_{*}\left(\Omega_{\mathfrak{U} / S}^{p}\right)$. For $p=\operatorname{dim} X$, we obtain the relative canonical sheaf $\omega_{\mathfrak{X} / S}$. In a similar vein, we have the relative tangent sheaf $\mathscr{T}_{\mathfrak{X} / S}=i_{*}\left(\mathscr{T}_{\mathfrak{W} / S}\right)$. If the deformation $\mathfrak{X} \rightarrow S$ is locally trivial, then after shrinking $\mathfrak{X}$ we may write $\mathfrak{X}=X \times S$. In this case, $\Omega_{\mathfrak{X} / S}^{[p]}=\operatorname{pr}_{1}^{*} \Omega_{X}^{[p]}$, where $\operatorname{pr}_{1}$ is the projection onto the first factor. In particular, the sheaf $\Omega_{\mathfrak{X} / S}^{[p]}$ is flat over $S$, and its restriction to the central fibre is $\Omega_{X}^{[p]}$.

Continuing to assume local triviality, let $g: \mathfrak{Y} \rightarrow \mathfrak{X}$ be a simultaneous resolution of $\mathfrak{X} \rightarrow S$. Then there is a natural push-forward map $g_{*} \Omega_{\mathfrak{Y} / S}^{p} \hookrightarrow \Omega_{\mathfrak{X} / S}^{[p]}$.

Proposition 2.7. If the central fibre $X$ has rational singularities, then the above map $g_{*} \Omega_{\mathfrak{Y} / S}^{p} \hookrightarrow \Omega_{\mathfrak{X} / S}^{[p]}$ is an isomorphism for any $p \geq 0$.

Proof. We employ induction on $n$, using the same notation and line of reasoning as in the proof of Lemma 2.6. Let $\bar{g}: Y \rightarrow X$ be the restriction of $g$ to the central fibre. For the start of induction, we need to know that $\bar{g}_{*} \Omega_{Y}^{p}=\Omega_{X}^{[p]}$, which follows from [KS19, Cor. 1.8] because $X$ has rational singularities. For the inductive step, tensor sequence (2.6.3) with the map in question. By local triviality, we obtain a commutative diagram

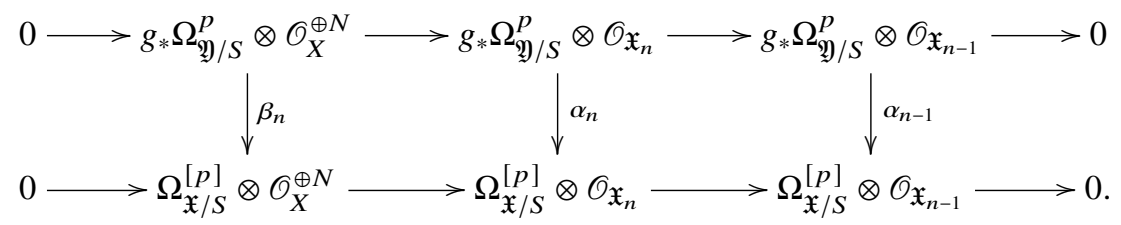

The map $\beta_{n}=\alpha_{0}^{\oplus N}$ is just a finite direct sum of copies of $\alpha_{0}$. In particular, it is an isomorphism. Likewise, $\alpha_{n-1}$ is an isomorphism by the inductive assumption. It now follows from the Five Lemma that $\alpha_{n}$ is also an isomorphism.

\section{Kähler metrics on singular spaces}

In [GK20], the first author associated to a Kähler metric on a singular complex space $X$ a cohomology class in the topological cohomology group $\mathrm{H}^{2}(X, \mathbb{R})$. Here, our focus will instead be on the (coherent) sheaf of Kähler differentials. The content of this section is probably well known to experts. Unfortunately, we have not been able to locate it in the published literature. The interested reader may check that all of the following is compatible with the usual notions if $X$ is smooth. 
Notation 2.8 (Pluriharmonic functions, [Var89, pp. 17, 23]). Let $X$ be a reduced complex space. We denote by $\mathscr{C}_{X, \mathbb{R}}^{\infty}$ the sheaf of smooth real-valued functions on $X$. Moreover, we denote by $\mathrm{PH}_{X, \mathbb{R}}$ the image of the real part map $\operatorname{Re}: \mathscr{O}_{X} \rightarrow \mathscr{C}_{X, \mathbb{R}}^{\infty}$, which is called the sheaf of real-valued pluriharmonic functions on $X$, and we set $\mathscr{K}_{X, \mathbb{R}}^{1}:=\mathscr{C}_{X, \mathbb{R}}^{\infty} / \mathrm{PH}_{X, \mathbb{R}}$.

The short exact sequence defining the sheaf $\mathscr{K}_{X, \mathbb{R}}^{1}$ gives rise to a connecting homomorphism

$$
\delta: \mathscr{K}_{X, \mathbb{R}}^{1}(X) \longrightarrow \mathrm{H}^{1}\left(X, \mathrm{PH}_{X, \mathbb{R}}\right) .
$$

Furthermore, because a holomorphic function with vanishing real part is locally constant, we have an isomorphism

$$
\mathrm{PH}_{X, \mathbb{R}} \cong \mathcal{O}_{X} / \underline{\mathbb{R}}_{X},
$$

where $\mathbb{R}_{X} \hookrightarrow \mathcal{O}_{X}$ is embedded via multiplication by i. Consequently we get an exterior differentiation map d: $\mathrm{PH}_{X, \mathbb{R}} \rightarrow \Omega_{X}^{1}$, where $\Omega_{X}^{1}$ is the sheaf of (universally finite) Kähler differentials [GK64, Satz 1.2]. This induces a map on cohomology

$$
\mathrm{d}: \mathrm{H}^{1}\left(X, \mathrm{PH}_{X, \mathbb{R}}\right) \longrightarrow \mathrm{H}^{1}\left(X, \Omega_{X}^{1}\right) .
$$

Composing, for any element $\kappa \in \mathscr{K}_{X, \mathbb{R}}^{1}(X)$, we get a class

$$
[\kappa]:=(\mathrm{d} \circ \delta)(\kappa) \in \mathrm{H}^{1}\left(X, \Omega_{X}^{1}\right) .
$$

Definition 2.9 (Kähler metrics, [Var89, pp. 23, 18]). Let $X$ be a reduced complex space.

(2.9.1) A Kähler metric on $X$ is an element $\omega$ of $\mathscr{K}_{X, \mathbb{R}}^{1}(X)$ that can be represented by a family $\left(U_{i}, \varphi_{i}\right)_{i \in I}$ such that $\varphi_{i}$ is a smooth strictly plurisubharmonic function on $U_{i}$ for all $i \in I$. That is, locally $\varphi_{i}$ is the restriction of a smooth strictly plurisubharmonic function on an open subset of $\mathbb{C}^{N_{i}}$ under a local embedding $U_{i} \hookrightarrow \mathbb{C}^{N_{i}}$.

(2.9.2) We say that $c \in \mathrm{H}^{1}\left(X, \Omega_{X}^{1}\right)$ is a Kähler class on $X$ if there exists a Kähler metric $\omega$ on $X$ such that $c=[\omega]$.

(2.9.3) We say that $X$ is Kähler if there exists a Kähler metric on $X$.

Of course, a Kähler class $[\omega]$ can be mapped further to $\mathrm{H}^{1}\left(X, \Omega_{X}^{[1]}\right)$, where $\Omega_{X}^{[1]}$ is the sheaf of reflexive differentials. Knowing that the class comes from the Kähler differentials, however, provides useful additional information: It shows that the class can be pulled back along arbitrary morphisms, even in situations where the extension theorem of [KS19] cannot be applied.

\section{A criterion for algebraic approximability}

The following theorem generalises the Green-Voisin criterion for algebraic approximability mentioned as step (B) in the Introduction. Let $X$ be a reduced complex space. To any class $c \in \mathrm{H}^{1}\left(X, \Omega_{X}^{1}\right)$, one can associate a linear map

$$
\alpha_{c}: \mathrm{H}^{1}\left(X, \mathscr{T}_{X}\right) \stackrel{c \cup-}{\longrightarrow} \mathrm{H}^{2}\left(X, \Omega_{X}^{1} \otimes \mathscr{T}_{X}\right) \stackrel{\text { contraction }}{\longrightarrow} \mathrm{H}^{2}\left(X, \mathcal{O}_{X}\right) .
$$

Theorem 3.1 (Approximation criterion for locally trivial deformations). Let $X$ be a compact Kähler space with rational singularities, and let $\pi: \mathfrak{X} \rightarrow S$ be a locally trivial deformation of $X$ over a smooth base S. Denote by

$$
\kappa(\pi): T_{0} S \longrightarrow \mathrm{H}^{1}\left(X, \mathscr{T}_{X}\right)
$$


the Kodaira-Spencer map of $\pi$. Assume that for some Kähler class $[\omega] \in \mathrm{H}^{1}\left(X, \Omega_{X}^{1}\right)$, the composition

$$
\alpha_{[\omega]} \circ \kappa(\pi): T_{0} S \longrightarrow \mathrm{H}^{2}\left(X, \mathcal{O}_{X}\right)
$$

is surjective. Then $\pi$ is a strong algebraic approximation of $X$.

Recall that the Kodaira-Spencer map $\kappa(\pi)$ for a locally trivial deformation of a singular space can be defined much in the same way as in the smooth case (cf. e.g., [Voi02, Sec. 9.1.2]. In particular, this implies that the left-hand square in the commutative diagram below actually is commutative.

Proof. Let $g: \mathfrak{Y} \rightarrow \mathfrak{X}$ be a simultaneous resolution of $\mathfrak{X} \rightarrow S$ with $\bar{g}: Y \rightarrow X$ the restriction to the central fibre, and let $f:=\pi \circ g: \mathfrak{Y} \rightarrow S$ be the composition. Furthermore, set $[\widetilde{\omega}]:=\left[\bar{g}^{*} \omega\right]$. Then there is the following commutative diagram:

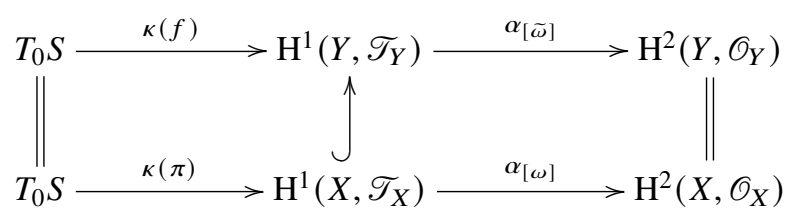

The vertical map on the left is the identity. The map in the middle comes from the fact that $\bar{g}_{*} \mathscr{T}_{Y}=\mathscr{T}_{X}$, which holds because $\bar{g}$ is the functorial resolution of $X$ (see [GK14, Thm. 4.2]). The vertical map on the right is an isomorphism because $X$ has rational singularities. Thus we see that

$$
\alpha_{[\widetilde{\omega}]} \circ \kappa(f): T_{0} S \longrightarrow \mathrm{H}^{2}\left(Y, \mathcal{O}_{Y}\right)
$$

is still surjective. Of course, $[\widetilde{\omega}]$ is not Kähler unless $X$ is already smooth. But because $g$ (and hence also $\bar{g}$ ) is a projective morphism, there exists a $\bar{g}$-ample line bundle $L$ on $Y$. For $\varepsilon>0$, consider the class $\left[\widetilde{\omega}_{\varepsilon}\right]:=[\widetilde{\omega}]+\varepsilon \cdot \mathrm{c}_{1}(L)$ and observe that $\alpha_{\left[\widetilde{\omega}_{\varepsilon}\right]}=\alpha_{[\widetilde{\omega}]}+\varepsilon \cdot \alpha_{\mathrm{c}_{1}(L)}$. Hence, we can choose $\varepsilon$ sufficiently small such that the map $\alpha_{\left[\widetilde{\omega}_{\varepsilon}\right]} \circ \kappa(f)$ remains surjective and the class $\left[\widetilde{\omega}_{\varepsilon}\right]$ is Kähler [Var89, Prop. II.1.3.1(vi)]. Thus, $f$ is a strong algebraic approximation of $Y$ by the Green-Voisin criterion [Voi03, Prop. 5.20].

This implies that $\pi$ is a strong algebraic approximation of $X$ : Because we assumed $X$ to be a compact Kähler space with rational singularities, we may shrink $S$ around its base point such that for every $t \in S$ the fibre $X_{t}$ is still Kähler [Bin83, Thm. 6.3] with rational singularities (by local triviality of $\pi$ or by [Elk78, Théorème 4]). If the fibre $Y_{t}$ for some $t \in S$ is projective, then $X_{t}$ is Moishezon and we conclude from [Nam02, Thm. 1.6] that $X_{t}$ is also projective.

\section{Surjectivity of cupping with a Kähler class}

In this section, we generalise step (C) from the Introduction to our singular setting. We then prove Theorem 1.2.

Theorem 4.1. Let $X$ be a normal compact complex space with klt singularities and $K_{X} \sim_{\mathbb{Q}} 0$. Then for any Kähler class $[\omega] \in \mathrm{H}^{1}\left(X, \Omega_{X}^{1}\right)$, the map $\alpha_{[\omega]}$ is surjective.

Proof. Let $\pi: X_{1} \rightarrow X$ be the index 1 cover of $X$. Then there is a finite (cyclic) group $G$ acting on $X_{1}$ such that $\pi$ is the quotient map. Because $K_{X} \sim \mathbb{Q} 0$, the map $\pi$ is quasi-étale and hence the $G$-action is free in codimension 1. Furthermore, $X_{1}$ has canonical (in particular, rational) singularities and $K_{X_{1}}$ is trivial [KM98, Prop. 5.20]. The pullback $\left[\pi^{*} \omega\right]$ is a $G$-invariant Kähler class on $X_{1}$ by [GK20, Prop. 3.5]. Assume for the moment that we can prove surjectivity of the $G$-equivariant map $\alpha_{\left[\pi^{*} \omega\right]}$. Then, because taking $G$-invariants is an exact functor [Mas99], the restriction to the invariant subspaces is 
also surjective. By [Gra18, Lemma 5.3], we obtain a commutative diagram

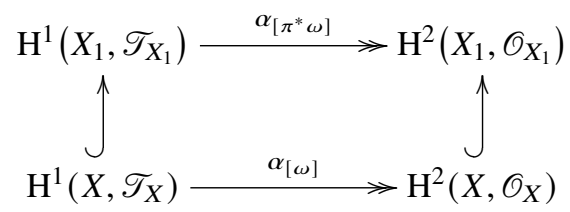

Thus, we may replace $X$ by $X_{1}$ and make the following additional assumption.

Additional Assumption 4.2. The canonical sheaf of $X$ is trivial, $\omega_{X} \cong \mathcal{O}_{X}$.

Fix a global generator $\sigma \in \mathrm{H}^{0}\left(X, \omega_{X}\right)$. Multiplication by $\sigma$ gives an isomorphism $\mathscr{O}_{X} \stackrel{\sim}{\rightarrow} \omega_{X}$, and contracting $\sigma$ by vector fields similarly yields $\mathscr{T}_{X} \stackrel{\sim}{\longrightarrow} \Omega_{X}^{[n-1]}$. We obtain the following diagram (whose vertical maps depend on the choice of $\sigma$ ):

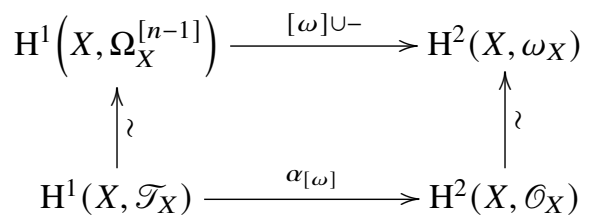

Claim 4.3. The above diagram is commutative.

Proof of Claim 4.3. We will compute in Čech cohomology with respect to a fixed Leray cover $\mathcal{U}:=$ $\left\{U_{i} \mid i \in I\right\}$ of $X$. Represent the given Kähler class $[\omega]$ by a cocycle $\left(\omega_{i j}\right)$ with $\omega_{i j} \in \Gamma\left(U_{i j}, \Omega_{X}^{1}\right)$ and pick an arbitrary class $\left[\left(v_{i j}\right)\right] \in \mathrm{H}^{1}\left(X, \mathscr{T}_{X}\right)$. Recall that quite generally, the cup product of two 1-cocycles $\left(\alpha_{i j}\right)$ and $\left(\beta_{i j}\right)$ is the 2-cocycle given by $\left(\alpha_{i j} \otimes \beta_{j k}\right)$. Hence, if we first move upwards and then to the right, we obtain the cocycle $\left(\omega_{i j} \wedge \iota_{v_{j k}}(\sigma)\right)$, where $\iota$ denotes contraction. If we go the other way around, we end up at $\left(\iota_{v_{j k}}\left(\omega_{i j}\right) \cdot \sigma\right)$. These two cocycles are cohomologous. In fact, they are equal on each open set $U_{i j k}$ because $\iota$ is an antiderivation and hence

$$
0=\iota_{v_{j k}}(\underbrace{\omega_{i j} \wedge \sigma}_{=0})=\iota_{v_{j k}}\left(\omega_{i j}\right) \wedge \sigma-\omega_{i j} \wedge \iota_{v_{j k}}(\sigma) .
$$

By Claim 4.3, it suffices to show that $\mathrm{H}^{1}\left(X, \Omega_{X}^{[n-1]}\right) \stackrel{[\omega] \cup-}{\longrightarrow} \mathrm{H}^{2}\left(X, \omega_{X}\right)$ is surjective. To this end, consider a strong projective resolution $f: Y \rightarrow X$ and the commutative diagram

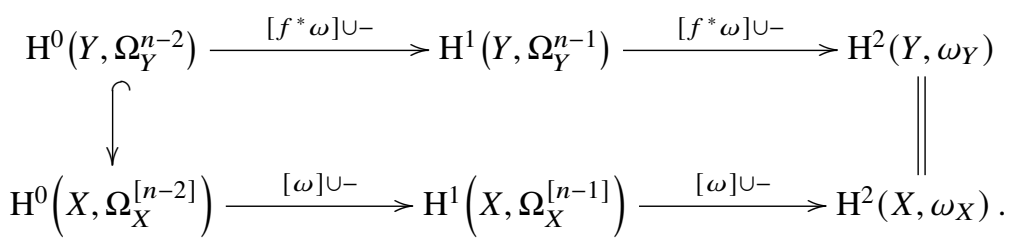

Claim 4.4. The vertical maps in the above diagram are isomorphisms.

Proof. Because $X$ has rational singularities, we have $f_{*} \omega_{Y}=\omega_{X}$ and the vertical map on the right is the natural map $\mathrm{H}^{2}\left(X, f_{*} \omega_{Y}\right) \rightarrow \mathrm{H}^{2}\left(Y, \omega_{Y}\right)$. This is an isomorphism because $R^{i} f_{*} \omega_{Y}=0$ for $i>0$ by Grauert-Riemenschneider vanishing [Tak85, Thm. 1]. The vertical map on the left is the pushforward map for reflexive differentials. It is an isomorphism by [KS19, Cor. 1.8], although we actually do not use this fact. 
Claim 4.5. The upper horizontal map $\mathrm{H}^{0}\left(Y, \Omega_{Y}^{n-2}\right) \stackrel{\left[f^{*} \omega\right]^{2} \cup-}{\longrightarrow} \mathrm{H}^{2}\left(Y, \omega_{Y}\right)$ in the above diagram is surjective.

Proof. By the hard Lefschetz theorem on the compact Kähler manifold $Y$ (or by the Hodge *-operator), the vector spaces $\mathrm{H}^{0}\left(Y, \Omega_{Y}^{n-2}\right)$ and $\mathrm{H}^{2}\left(Y, \omega_{Y}\right)$ have the same dimension. It is therefore sufficient to prove injectivity of the map in question. So let $\alpha \in \mathrm{H}^{0}\left(Y, \Omega_{Y}^{n-2}\right) \backslash\{0\}$ be a nonzero holomorphic $(n-2)$-form. We claim that

$$
\underbrace{\mathrm{i}^{n-2}(-1)^{\frac{(n-2)(n-3)}{2}}}_{=\mathrm{i}^{n^{2}}} \int_{Y} \alpha \wedge \bar{\alpha} \wedge f^{*} \omega^{2}>0 .
$$

Indeed, set $E=\operatorname{Exc}(f)$ and fix a volume form vol on $Y$ that on $Y \backslash E$ is compatible with the orientation induced by $f^{*} \omega$. Then outside of $E_{\alpha}:=E \cup\{\alpha=0\}$, the integrand in (4.5.1) is a strictly positive multiple of vol by the (pointwise) Hodge-Riemann bilinear relations [Huy05, Cor. 1.2.36]. Being an analytic subset of lower dimension, $E_{\alpha}$ has measure 0 . Inequality (4.5.1) follows.

By Stokes' theorem and (4.5.1), the form $\alpha \wedge \bar{\alpha} \wedge f^{*} \omega^{2}$ cannot be d-exact. On the other hand, the global holomorphic form $\alpha$ is d-closed, so $\mathrm{d} \bar{\alpha}=\overline{\mathrm{d} \alpha}=0$. Thus, $\alpha \wedge f^{*} \omega^{2}$ is also not d-exact. Equivalently, by the $\partial \bar{\partial}$-lemma, $\alpha \wedge f^{*} \omega^{2}$ is not $\bar{\partial}$-exact. Interpreting $\mathrm{H}^{2}\left(Y, \omega_{Y}\right)$ as a Dolbeault cohomology group, the latter form represents the class $\left[f^{*} \omega\right]^{2} \cup \alpha$, which is therefore nonzero.

The desired surjectivity of the lower right map in diagram (4.3.1) now follows from Claim 4.5 and a simple diagram chase, ending the proof of Theorem 4.1.

Remark. Theorem 4.1 continues to hold for $X$ with rational singularities as long as $\omega_{X}$ is trivial. However, taking the index 1 cover in general does not preserve rationality and then the proof has some problems. An easy example is given by the (rational) cone over an Enriques surface, which has a quasiétale cover by the (nonrational) cone over the corresponding K3 surface.

\section{Proof of Theorem 1.2}

Let $X$ be a normal compact Kähler space with klt singularities and $K_{X} \sim_{\mathbb{Q}} 0$. We assume that the base of the semi-universal locally trivial deformation $\pi: \mathfrak{X} \rightarrow \operatorname{Def}^{\text {lt }}(X)$ is smooth. Let $[\omega] \in \mathrm{H}^{1}\left(X, \Omega_{X}^{1}\right)$ be a Kähler class on $X$. By Theorem 4.1, cupping with $[\omega]$ induces a surjective map $\alpha_{[\omega]}: \mathrm{H}^{1}\left(X, \mathscr{T}_{X}\right) \longrightarrow$ $\mathrm{H}^{2}\left(X, \mathscr{O}_{X}\right)$. By semi-universality of $\pi$, the Kodaira-Spencer map $\kappa(\pi): T_{0} \operatorname{Def}^{\text {lt }}(X) \longrightarrow \mathrm{H}^{1}\left(X, \mathscr{T}_{X}\right)$ is an isomorphism and the composition

$$
\alpha_{[\omega]} \circ \kappa(\pi): T_{0} \operatorname{Def}^{\mathrm{lt}}(X) \longrightarrow \mathrm{H}^{2}\left(X, \mathcal{O}_{X}\right)
$$

is surjective. By Theorem 3.1, it follows that $\pi$ is a strong algebraic approximation of $X$.

\section{Locally trivial unobstructedness}

\section{A. $T^{1}$-lifting principle for locally trivial deformations}

Let $X$ be a normal compact complex space. We want to prove smoothness of $\operatorname{Def}^{\text {lt }}(X)$ under the assumptions of Theorems 1.4 and 1.5. In both cases, our aim is to apply Kawamata-Ran's $T^{1}$-lifting principle [Ran92, Kaw92, Kaw97, FM99]. For this, let $f: \mathfrak{X} \rightarrow S=\operatorname{Spec} A$ be an infinitesimal locally trivial deformation of $X$, let $S^{\prime}=\operatorname{Spec} A^{\prime} \subset S$ be a closed subscheme and let $f^{\prime}: \mathfrak{X}^{\prime} \rightarrow S^{\prime}$ be the induced deformation. Then the $T^{1}$-lifting property boils down to surjectivity of the canonical map

$$
\mathrm{H}^{1}\left(\mathfrak{X}, \mathscr{T}_{\mathfrak{X} / S}\right) \longrightarrow \mathrm{H}^{1}\left(\mathfrak{X}^{\prime}, \mathscr{T}_{\mathfrak{X}^{\prime} / S^{\prime}}\right) .
$$




\section{B. Proof of Theorem 1.4}

With notation as above, assume additionally that $\mathrm{H}^{2}\left(X, \mathscr{T}_{X}\right)=0$. To apply the $T^{1}$-lifting principle, it actually suffices to check surjectivity of (5.1.1) in the special case $A=A_{n+1}$ and $A^{\prime}=A_{n}$, where $A_{n}:=\mathbb{C}[t] /\left(t^{n+1}\right)$ and the map $A \rightarrow A^{\prime}$ is the natural one [Kaw92, Thm. 1]. For every given $n \in \mathbb{N}_{0}$, the short exact sequence

$$
0 \longrightarrow\left(t^{n+1}\right) \longrightarrow A_{n+1} \longrightarrow A_{n} \longrightarrow 0
$$

induces by flatness (cf. the proof of Lemma 2.6)

$$
0 \longrightarrow \mathcal{O}_{X} \longrightarrow \mathcal{O}_{\mathfrak{X}} \longrightarrow \mathcal{O}_{\mathfrak{X}^{\prime}} \longrightarrow 0,
$$

which, after tensorising with $\mathscr{T}_{\mathfrak{X} / S}$, yields

$$
0 \longrightarrow \mathscr{T}_{X} \longrightarrow \mathscr{T}_{\mathfrak{X} / S} \longrightarrow \mathscr{T}_{\mathfrak{X}^{\prime} / S^{\prime}} \longrightarrow 0 .
$$

For exactness in the middle and on the right, see the proof of Proposition 2.7. Injectivity on the left is best seen using a local trivialisation $\mathfrak{X} \cong X \times S$ and the description of $\mathscr{T}_{\mathfrak{X} / S}$ as $p^{*} \mathscr{T}_{X}$, where $p: \mathfrak{X} \rightarrow X$ is the first projection. The long exact sequence associated to (5.1.2), combined with the assumption $\mathrm{H}^{2}\left(X, \mathscr{T}_{X}\right)=0$, now immediately yields surjectivity of the map (5.1.1), completing the proof.

\section{C. Proof of Theorem 1.5}

We drop the assumption $\mathrm{H}^{2}\left(X, \mathscr{T}_{X}\right)=0$ and instead assume $X$ to be Kähler with rational singularities and $\omega_{X} \cong \mathcal{O}_{X}$. By Lemma 5.1 below, $\omega_{\mathfrak{X} / S} \cong \mathcal{O}_{\mathfrak{X}}$. By contraction, we obtain an isomorphism $\mathscr{T}_{\mathfrak{X} / S} \cong$ $\mathscr{T}_{\mathfrak{X} / S} \otimes \omega_{\mathfrak{X} / S}=\Omega_{\mathfrak{X} / S}^{[n-1]}$. Hence, the surjectivity of (5.1.1) is equivalent to the surjectivity of

$$
\mathrm{H}^{1}\left(\mathfrak{X}, \Omega_{\mathfrak{X} / S}^{[n-1]}\right) \longrightarrow \mathrm{H}^{1}\left(\mathfrak{X}^{\prime}, \Omega_{\mathfrak{X}^{\prime} / S^{\prime}}^{[n-1]}\right) .
$$

We will show that $R^{1} f_{*} \Omega_{\mathfrak{X} / S}^{[n-1]}$ is locally free and commutes with base change. In this case, (5.1.3) simply is the canonical map

$$
\mathrm{H}^{1}\left(\mathfrak{X}, \Omega_{\mathfrak{X} / S}^{[n-1]}\right) \longrightarrow \mathrm{H}^{1}\left(\mathfrak{X}, \Omega_{\mathfrak{X} / S}^{[n-1]}\right) \otimes_{A} A^{\prime},
$$

which is surjective because $A \rightarrow A^{\prime}$ is.

Lemma 5.1. Let $f: \mathfrak{X} \rightarrow S=\operatorname{Spec} A$ be an infinitesimal locally trivial deformation of a normal compact Kähler space $X$ with rational singularities and $\omega_{X} \cong \mathcal{O}_{X}$. Then the relative canonical sheaf $\omega_{\mathfrak{X} / S}$ is also trivial.

Proof. Let $g: \mathfrak{Y} \rightarrow \mathfrak{X}$ be a simultaneous resolution of $\mathfrak{X} \rightarrow S$. Then the composition $\pi: \mathfrak{Y} \rightarrow S$ is a deformation of the compact Kähler manifold $Y$, where $\bar{g}: Y \rightarrow X$ is the functorial resolution of the central fibre $X$.

By [De168, Théorème 5.5], the sheaf $\pi_{*} \omega_{\mathfrak{Y} / S}$ is locally free (hence free, because $A$ is local). Because it also satisfies base change, its rank may be calculated from the central fibre, $h^{0}\left(Y, \omega_{Y}\right)=h^{0}\left(X, \omega_{X}\right)=1$. Because $\pi_{*}(-)=f_{*} g_{*}(-)$, we thus obtain from Proposition 2.7 that $f_{*} \omega_{\mathfrak{X} / S} \cong \mathcal{O}_{S}$. Pick a global section $\sigma$ of $\omega \mathfrak{X} / S$ that under the identification

$$
\mathrm{H}^{0}\left(\mathfrak{X}, \omega_{\mathfrak{X} / S}\right)=\mathrm{H}^{0}\left(S, f_{*} \omega_{\mathfrak{X} / S}\right) \cong \mathrm{H}^{0}\left(S, \mathcal{O}_{S}\right)=A
$$

corresponds to an element of $A^{\times}$; that is, to a unit of $A$. This gives rise to a map $\sigma: \mathcal{O}_{\mathfrak{X}} \longrightarrow \omega_{\mathfrak{X} / S}$, whose restriction to the central fibre is an isomorphism. By the same argument as in the proof of Proposition 2.7, we may deduce from this that $\sigma$ itself is an isomorphism. This ends the proof. 
Recall that we are currently proving Theorem 1.5 and hence we also assume that the reflexive Poincaré lemma holds on $X$ and that the corresponding spectral sequence (1.4.1) degenerates at the first page in degree $n$.

Claim 5.2 (Relative Poincaré lemma on $\mathfrak{X}$ over $S$ ). The complex $\Omega_{\mathfrak{X} / S}^{[\bullet]}$ is a resolution of the constant sheaf $\underline{A}_{\mathfrak{X}}$.

Proof. The claim is local on $\mathfrak{X}$. By local triviality of $\mathfrak{X} \rightarrow S$, we may therefore assume that $\mathfrak{X} \cong X \times S$. By assumption, $\Omega_{X}^{[\bullet]}$ is exact in degree $\geq 1$. Because the projection $p: X \times S \rightarrow X$ is flat, $p^{*} \Omega_{X}^{[\bullet]}=\Omega_{\mathfrak{X} / S}^{[\bullet]}$ is also exact in degree $\geq 1$. The kernel of $\mathcal{O}_{\mathfrak{X}} \rightarrow \Omega_{\mathfrak{X} / S}^{[1]}$ consists of those functions that are pulled back from $S$; that is, the sheaf $f^{-1} \mathcal{O}_{S}$. Bcause $\mathcal{O}_{S}=\underline{A}_{S}$, we have $f^{-1} \mathcal{O}_{S}=\underline{A}_{\mathfrak{X}}$.

By flatness of $\Omega_{\mathfrak{X} / S}^{[p]}$ over $S$ and by [BS76, Ch. III, Thm. 4.1], there are bounded complexes of finitely generated free $A$-modules $L_{p}^{\bullet}, 1 \leq p \leq n$, such that

$$
R^{q} f_{*}\left(\Omega_{\mathfrak{X} / S}^{[p]} \otimes f^{*} Q\right)=\mathrm{H}^{q}\left(L_{p}^{\bullet} \otimes Q\right)
$$

for any $q \geq 0$ and any finitely generated $A$-module $Q$. We will apply these statements to $Q=A / \mathrm{m}=\mathbb{C}$. Let $\ell(-)$ denote the length of an $A$-module and recall that this is additive on short exact sequences. By [Del68, (3.5)], we have the following inequality:

$$
\ell\left(\mathrm{H}^{q}\left(L_{p}^{\bullet}\right)\right) \leq \ell(A) \cdot \operatorname{dim}_{\mathbb{C}} \mathrm{H}^{q}\left(L_{p}^{\bullet} \otimes \mathbb{C}\right) \quad \text { for all } q \geq 0 \text { and } 1 \leq p \leq n .
$$

Furthermore, as soon as we can show that equality holds for $(p, q)=(n-1,1)$, it follows from [Del68, (3.5)] again that $R^{1} f_{*} \Omega_{\mathfrak{X} / S}^{[n-1]}$ is locally free and commutes with base change, and the proof of Theorem 1.5 will be finished. In other words, using (5.2.1), we have to show that

$$
\ell\left(R^{1} f_{*} \Omega_{\mathfrak{X} / S}^{[n-1]}\right)=\ell(A) \cdot \operatorname{dim}_{\mathbb{C}} \mathrm{H}^{1}\left(X, \Omega_{X}^{[n-1]}\right) .
$$

To this end, consider the following chain of inequalities:

$$
\begin{aligned}
\ell\left(R^{n} f_{*} \Omega_{\mathfrak{X} / S}^{[\bullet]}\right) & \leq \sum_{p+q=n} \ell\left(R^{q} f_{*} \Omega_{\mathfrak{X} / S}^{[p]}\right) & & \\
& \leq \ell(A) \cdot \sum_{p+q=n} h^{q}\left(X, \Omega_{X}^{[p]}\right) & & \text { by (5.2.1) and (5.2.2) } \\
& \leq \ell(A) \cdot h^{n}(X, \mathbb{C}) & & \text { partial degeneration of }(1.4 .1) \\
& =\ell\left(\mathrm{H}^{n}\left(\mathfrak{X}, \underline{A}_{\mathfrak{X}}\right)\right) & & \text { universal coefficient theorem. }
\end{aligned}
$$

The first inequality comes from the spectral sequence associated to the stupid filtration on the complex $\Omega_{\mathfrak{X} / S}^{[\bullet]}$. By Claim 5.2 we have $R^{n} f_{*} \Omega_{\mathfrak{X} / S}^{[\bullet]} \cong \mathrm{H}^{n}\left(\mathfrak{X}, \underline{A}_{\mathfrak{X}}\right)$, and therefore equality holds everywhere in the above chain of inequalities. In particular, for $(p, q)=(n-1,1)$ we get the desired equality (5.2.3).

\section{D. Proof of Corollary 1.6}

Let $X$ be a normal compact Kähler space with quotient or toroidal singularities and $K_{X} \sim_{\mathbb{Q}} 0$. Consider the index 1 cover $X_{1} \rightarrow X$. Then $K_{X_{1}} \sim 0$ and $X_{1}$ has the same kind of singularities by Proposition 5.3. By Proposition 5.4, smoothness of $\operatorname{Def}^{\text {lt }}\left(X_{1}\right)$ implies the same property for $X$.

Proposition 5.3. Let $X$ be a normal complex space such that $K_{X} \sim_{\mathbb{Q}} 0$, and let $X_{1} \rightarrow X$ be the index 1 cover. If $X$ has only quotient (respectively toroidal) singularities, then the same is true for $X_{1}$. 
Proof. For quotient singularities, the statement is well known and can in fact easily be deduced from [CGGN20, Lemma 2.9]. In the toroidal case, we will freely use standard facts and notation from toric geometry as expounded, for example, in [CLS11]. Assume now that $X$ has toroidal singularities, and fix $x \in X$. Then there is a Euclidean open neighbourhood $x \in U \subset X$ and an isomorphism $\varphi: U \stackrel{\sim}{\longrightarrow} V \subset X^{\prime}$, where $X^{\prime}$ is toric and $\varphi(x)$ is a torus fixed point. By Sumihiro's theorem, we may shrink $X^{\prime}$ around $\varphi(x)$ and assume that $X^{\prime}$ is affine and $K_{X^{\prime}} \sim \mathbb{Q} 0$. In the remainder of the proof, we will explain the toric construction of the index 1 cover of $X^{\prime}$. We abuse notation and replace $X$ by $X^{\prime}$.

So let $N, M$ be dual lattices and let $\sigma \subset N_{\mathbb{R}}$ be a strictly convex rational polyhedral cone with $X=U_{\sigma, N}=\operatorname{Spec} \mathbb{C}\left[\sigma^{\vee} \cap M\right]$. Let $\sigma(1)$ be the set of extremal rays of $\sigma$ and $u_{\rho}$ the minimal generator of $\rho \cap N$, for any $\rho \in \sigma(1)$. Because $X$ is in particular $\mathbb{Q}$-Gorenstein, there exists $m_{0} \in M_{\mathbb{Q}}$ such that $\left\langle m_{0}, u_{\rho}\right\rangle=1$ for all $\rho \in \sigma(1)$. If $r$ is the index of $K_{X}$, then $r m_{0} \in M$ and $r$ is the smallest positive integer with this property.

Set $\widetilde{N}:=m_{0}^{-1}(\mathbb{Z}) \subset N$, considering $m_{0}$ as a map $N \rightarrow \mathbb{Z} \cdot \frac{1}{r} \subset \mathbb{Q}$. Then $\widetilde{N}$ contains the set $\left\{u_{\rho} \mid \rho \in \sigma(1)\right\}$ and $N / \widetilde{N} \cong \mathbb{Z} \frac{1}{r} / \mathbb{Z} \cong \mathbb{Z} / r \mathbb{Z}$ is finite cyclic. In particular, $\widetilde{N}_{\mathbb{R}}=N_{\mathbb{R}}, M \subset \widetilde{M}$ has finite index and $M_{\mathbb{Q}}=\widetilde{M}_{\mathbb{Q}}$. The inclusion $\sigma^{\vee} \cap M \subset \sigma^{\vee} \cap \widetilde{M}$ gives rise to a finite cyclic degree $r$ Galois cover

$$
\pi: X_{1}:=U_{\sigma, \widetilde{N}}=\operatorname{Spec} \mathbb{C}\left[\sigma^{\vee} \cap \widetilde{M}\right] \longrightarrow X .
$$

Note that when passing from $N$ to $\widetilde{N}$, neither $\sigma(1)$ nor the individual $u_{\rho}$ change. Therefore, because $m_{0} \in \widetilde{M} \subset \widetilde{M}_{\mathbb{Q}}$ and we still have $\left\langle m_{0}, u_{\rho}\right\rangle=1$, it follows that $K_{X_{1}}$ is Cartier. Better still, $K_{X_{1}} \sim 0$ as $X_{1}$ is affine.

It remains to show that $\pi$ is quasi-étale. The $T_{N}$-invariant prime divisors on $X$ are in bijection with the $T_{\widetilde{N}}$-invariant prime divisors on $X_{1}$ because both are in bijection with $\sigma(1)$ by the orbit-cone correspondence. Recall that this correspondence sends $\rho$ to $D_{\rho, X}:=$ the closure of the orbit of $\gamma_{\rho, X}$, where $\gamma_{\rho, X}$ is the $t \rightarrow 0$ limit of the 1-parameter subgroup $\mathbb{C}^{*} \rightarrow X$ defined by $u_{\rho}$, and analogously for $D_{\rho, X_{1}}$. Because $\pi\left(\gamma_{\rho, X_{1}}\right)=\gamma_{\rho, X}$, it follows that $\pi\left(D_{\rho, X_{1}}\right)=D_{\rho, X}$ set-theoretically. Even better, because $\pi$ is surjective and the number of invariant divisors on $X$ and on $X_{1}$ is the same, we must have $\pi^{-1}\left(D_{\rho, X}\right)=D_{\rho, X_{1}}$ set-theoretically. In other words, $\pi^{*}\left(D_{\rho, X}\right)=\mu_{\rho} \cdot D_{\rho, X_{1}}$ as divisors, and we need to show that all $\mu_{\rho}=1$.

Now the divisor of the character $\chi$ corresponding to $r m_{0} \in M$ is

$$
\operatorname{div}(\chi)=\sum_{\rho \in \sigma(1)} r D_{\rho, X},
$$

and using that $\operatorname{div}\left(\pi^{*} \chi\right)=\pi^{*} \operatorname{div}(\chi)$, we get

$$
\sum_{\rho \in \sigma(1)} r D_{\rho, X_{1}}=\sum_{\rho \in \sigma(1)} r \mu_{\rho} D_{\rho, X_{1}} .
$$

Comparing coefficients, it follows that $\mu_{\rho}=1$ for all $\rho \in \sigma(1)$, as desired.

Proposition 5.4. Let $Y$ be a normal compact complex space, and let $G$ be a finite group acting effectively on $Y$. Assume that $\operatorname{Def}^{\mathrm{lt}}(Y)$ is smooth. Then there is a locally trivial deformation $\pi: \mathfrak{Y} \rightarrow \Delta$ of $Y$ over a smooth base $0 \in \Delta$ such that:

(5.4.1) The group $G$ acts on $\mathfrak{Y}$ in a fibre-preserving way, extending the given action $G G Y$.

(5.4.2) The Kodaira-Spencer map $\kappa(\pi): T_{0} \Delta \rightarrow \mathrm{H}^{1}\left(Y, \mathscr{T}_{Y}\right)$ is an isomorphism onto $\mathrm{H}^{1}\left(Y, \mathscr{T}_{Y}\right)^{G} \subset$ $\mathrm{H}^{1}\left(Y, \mathscr{T}_{Y}\right)$.

Put $X:=Y / G$ and $\varphi: \mathfrak{X}:=\mathfrak{Y} / G \rightarrow \Delta$. Then:

(5.4.3) The map $\varphi$ is a locally trivial deformation of $X$. 
(5.4.4) If the action of $G$ on $Y$ is free in codimension 1, then $\varphi$ is the semi-universal locally trivial deformation of $X$. In particular, $\operatorname{Def}^{\mathrm{lt}}(X)$ is smooth in this case.

Proof. In the case that $Y$ is smooth, this is [Gra18, Prop. 6.1, Prop. 6.2]. For general $Y$, the proof still works because we only consider locally trivial deformations of $Y$.

By the above argument, we may replace $X$ by $X_{1}$ and assume $K_{X} \sim 0$. Our aim is to apply Theorem 1.5, so we first need to know that the reflexive Poincaré lemma holds on $X$. For quotient singularities, this is [Ste77, Cor. 1.9]. In the toroidal case, we use [Dan91, Lemma 2.3] instead. Note that these references work in an algebraic context; that is, $X$ is assumed to be an algebraic variety. This, however, is not a problem because the claim and its proof are analytically local.

We also need to know that (1.4.1) degenerates at $E_{1}$ in degree $n=\operatorname{dim} X$. In our situation, this degeneration even holds in any degree: In the orbifold case, apply [Ste77, Thm. 1.12], and for toroidal spaces, use [Dan91, Thm. 3.4.a)]. Again, the results are stated only for algebraic varieties. But a close inspection of the proofs reveals that this assumption is, in both cases, only invoked once in a substantial way: $X$ has a resolution of singularities on which (1.4.1) degenerates. Because this still holds true for compact Kähler spaces, degeneration remains valid in our setting.

It is well known that quotient singularities as well as $\mathbb{Q}$-Gorenstein toroidal singularities are klt [KM98, Prop. 5.13], [CLS11, Cor. 11.4.25(b)]. In particular, they are rational [KM98, Thm. 5.22]. Corollary 1.6 is thus a consequence of Theorem 1.5.

\section{E. Proof of Corollary 1.7}

This immediately follows from Theorems 1.2 and 1.4 and Corollary 1.6.

Acknowledgments. Patrick Graf thanks Professor E. Sernesi for helpful discussions about deformation theory, in particular about Theorem 1.4. Both authors thank the anonymous referee for careful proofreading and several suggestions that improved our exposition. Patrick Graf was partially supported by a DFG Research Fellowship. Martin Schwald was partially supported by the DFG Collaborative Research Centre SFB/TR 45. This publication was funded by the German Research Foundation (DFG) and the University of Bayreuth in the funding programme Open Access Publishing.

Conflict of Interest: None.

\section{References}

[BGL20] B. Bakker, H. Guenancia and C. Lehn, 'Algebraic approximation and the decomposition theorem for Kähler Calabi-Yau varieties', Preprint, 2020, arXiv:2012.00441.

[BL19] B. Bakker and C. Lehn, 'The global moduli theory of symplectic varieties', Preprint, 2019, arXiv:1812.09748.

[BS76] C. Bănică and O. Stănăşilă, Algebraic Methods in the Global Theory of Complex Spaces (John Wiley \& Sons, London-New York-Sydney, 1976).

[Bin83] J. Bingener, 'On deformations of Kähler spaces. I', Math. Z. 182(4) (1983), 505-535.

[Bog78] F. A. Bogomolov, 'Hamiltonian Kählerian manifolds', Dokl. Akad. Nauk SSSR 243(5) (1978), 1101-1104.

[Cam04] F. Campana, 'Orbifoldes à première classe de Chern nulle', The Fano Conference' (University of Torino, Turin, Italy, 2004), pp. 339-351.

[CGGN20] B. Claudon, P. Graf, H. Guenancia and P. Naumann, 'Kähler spaces with zero first Chern class: Bochner principle, fundamental groups, and the Kodaira problem', Preprint, 2020, arXiv:2008.13008.

[CHL19] B. Claudon, A. Höring and H.-Y. Lin, 'The fundamental group of compact Kähler threefolds', Geom. Topol. 23 (2019), 3233-3271.

[CLS11] D. A. Cox, J. B. Little and H. K. Schenck, Toric Varieties, Graduate Studies in Mathematics, Vol. 124 (American Mathematical Society, Providence, RI, 2011).

[Dan91] V. I. Danilov, 'De Rham complex on toroidal variety', in Algebraic Geometry (Chicago, IL, 1989), Lecture Notes in Mathematics, Vol. 1479 (Springer, Berlin, 1991), pp. 26-38.

[De168] P. Deligne, 'Théorème de Lefschetz et critères de dégénérescence de suites spectrales', Publ. Math. de l'I.H.É.S. (1968), no. 35, 259-278.

[Elk78] R. Elkik, 'Singularités rationnelles et déformations', Invent. Math. 47(2) (1978), 139-147.

[FM99] B. Fantechi and M. Manetti, 'On the $\mathrm{T}^{1}$-lifting theorem', J. Algebraic Geom. 8(1) (1999), 31-39.

[FK87] H. Flenner and S. Kosarew, 'On locally trivial deformations', Publ. RIMS Kyoto 23 (1987), 627-665. 
[Gra18] P. Graf, 'Algebraic approximation of Kähler threefolds of Kodaira dimension zero', Math. Ann. 371 (2018), 487516.

[Gra21] P. Graf, 'A decomposition theorem for singular Kähler spaces with trivial first Chern class of dimension at most four', Preprint, 2021, arXiv:2101.06764.

[GK20] P. Graf and T. Kirschner, 'Finite quotients of three-dimensional complex tori', Ann. Inst. Fourier (Grenoble) 70(2) (2020), 881-914.

[GK14] P. Graf and S. J. Kovács, 'An optimal extension theorem for 1-forms and the Lipman-Zariski conjecture', Documenta Math. 19 (2014), 815-830.

[GS20] P. Graf and M. Schwald, 'On the Kodaira problem for uniruled Kähler spaces', Ark. Mat. 58(2) (2020), $267-284$.

[GK64] H. Grauert and H. Kerner, 'Deformationen von Singularitäten komplexer Räume', Math. Ann. 153 (1964), $236-260$.

[Gro97] M. Gross, 'The deformation space of Calabi-Yau n-folds with canonical singularities can be obstructed', in Mirror Symmetry, II, AMS/IP Studies in Advanced Mathematics, Vol. 1 (American Mathematical Society, Providence, RI, 1997), pp. 401-411.

[Huy05] D. Huybrechts, Complex Geometry (Springer-Verlag, Berlin, 2005).

[Jör14] C. Jörder, On the Poincaré Lemma for Reflexive Differential Forms (PhD Thesis, Albert-Ludwigs-Universität Freiburg im Breisgau, 2014). URL: https://freidok.uni-freiburg.de/data/9438.

[Kaw92] Y. Kawamata, 'Unobstructed deformations. A remark on a paper of Z. Ran', J. Algebraic Geom. 1(2) (1992), 183-190.

[Kaw97] Y. Kawamata, 'Erratum on: "Unobstructed deformations. A remark on a paper of Z. Ran"', J. Algebraic Geom. 6(4) (1997), 803-804.

[KS19] S. Kebekus and C. Schnell, 'Extending holomorphic forms from the regular locus of a complex space to a resolution of singularities', Preprint, 2019, arXiv:1811.03644.

[Kod63] K. Kodaira, 'On compact analytic surfaces, III', Ann. Math. 78(1) (1963), 1-40.

[KNS58] K. Kodaira, L. Nirenberg and D. C. Spencer, 'On the existence of deformations of complex analytic structures', Ann. Math. (2) 68 (1958), 450-459.

[Kol07] J. Kollár, Lectures on Resolution of Singularities, Annals of Mathematics Studies, Vol. 166 (Princeton University Press, Princeton, NJ, 2007).

[KM98] J. Kollár and S. Mori, Birational Geometry of Algebraic Varieties, Cambridge Tracts in Mathematics, Vol. 134 (Cambridge University Press, Cambridge, UK, 1998).

[Lin18a] H.-Y. Lin, 'Algebraic approximations of compact Kähler threefolds', Preprint, 2018, arXiv:1710.01083.

[Lin18b] H.-Y. Lin, 'Algebraic approximations of fibrations in abelian varieties over a curve', Preprint, 2018, arXiv:1612.09271.

[Mas99] H. Maschke, 'Beweis des Satzes, dass diejenigen endlichen linearen Substitutionsgruppen, in welchen einige durchgehends verschwindende Coefficienten auftreten, intransitiv sind', Math. Ann. 52(2-3) (1899), 363-368.

[Nam02] Y. Namikawa, 'Projectivity criterion of Moishezon spaces and density of projective symplectic varieties', Int. J. Math. 13(2) (2002), 125-135.

[Ran92] Z. Ran, 'Deformations of manifolds with torsion or negative canonical bundle', J. Algebraic Geom. 1(2) (1992), 279-291.

[Ser06] E. Sernesi, Deformations of Algebraic Schemes, Grundlehren der Mathematischen Wissenschaften, Vol. 334 (Springer-Verlag, Berlin, 2006).

[Ste77] J. H. M. Steenbrink, 'Mixed Hodge structure on the vanishing cohomology, Real and complex singularities', in Proc. Ninth Nordic Summer School/NAVF Sympos. Math., Oslo, 1976 (Sijthoff and Noordhoff , Alphen aan den Rijn, Netherlands, 1977), pp. 525-563.

[Tak85] K. Takegoshi, 'Relative vanishing theorems in analytic spaces', Duke Math. J. 52(1) (1985), 273-279.

[Tia87] G. Tian, 'Smoothness of the universal deformation space of compact Calabi-Yau manifolds and its Petersson-Weil metric', in Mathematical Aspects of String Theory (San Diego, Calif., 1986), Adv. Ser. Math. Phys., Vol. 1 (World Scientific, Singapore, 1987), pp. 629-646.

[Tod89] A. N. Todorov, 'The Weil-Petersson geometry of the moduli space of SU ( $\mathrm{n} \geq 3$ )(Calabi-Yau) manifolds. I', Comm. Math. Phys. 126(2) (1989), 325-346.

[Var89] J. Varouchas, 'Kähler spaces and proper open morphisms', Math. Ann. 283(1) (1989), 13-52.

[Voi02] C. Voisin, Hodge Theory and Complex Algebraic Geometry I, Cambridge Studies in Advanced Mathematics, Vol. 76 (Cambridge University Press, Cambridge, UK, 2002).

[Voi03] C. Voisin, Hodge Theory and Complex Algebraic Geometry II, Cambridge Studies in Advanced Mathematics, Vol. 77 (Cambridge University Press, 2003).

[Voi04] C. Voisin, 'On the homotopy types of compact Kähler and complex projective manifolds', Invent. Math. 157 (2004), 329-343.

[Voi06] C. Voisin, 'On the homotopy types of Kähler manifolds and the birational Kodaira problem', J. Diff. Geom. 72 (2006), 43-71. 